\title{
Ultralow threshold graded-index separate-confinement heterostructure single quantum well (Al,Ga)As lasers
}

\author{
P.L. Derry, H.Z. Chen, H. Morkoç, and A. Yariv \\ California Institute of Technology, Pasadena, California 91125 \\ K. Y. Lau, N. Bar-Chaim, K. Lee, and J. Rosenberg \\ ORTEL Corporation, Alhambra, California 91803
}

(Received 9 September 1987; accepted 17 November 1987)

\begin{abstract}
Broad area graded-index separate-confinement heterostructure single quantum well lasers grown by molecular-beam epitaxy (MBE) with threshold current density as low as $93 \mathrm{~A} / \mathrm{cm}^{2}(520 \mu \mathrm{m}$ long) have been fabricated. Buried lasers formed from similarly structured MBE material with liquid phase epitaxy regrowth had threshold currents at submilliampere levels when high reflectivity coatings were applied to the end facets. A cw threshold current of $0.55 \mathrm{~mA}$ was obtained for a laser with facet refiectivities of $\sim 80 \%$, a cavity length of $120 \mu \mathrm{m}$, and an active region stripe width of $1 \mu \mathrm{m}$. These devices driven directly with logic level signals have switch-on delays $<50$ ps without any current prebias. Such lasers permit fully on-ofi switching while at the same time obviating the need for bias monitoring and feedback control.
\end{abstract}

Semiconductor lasers are being considered for future generations of supercomputers. The number of lasers that may be involved is so large that a major premium is placed on a reduction of the threshold current of such lasers. Lasers which can be modulated at high speed without current prebias and a feedback circuit are also highly desirable for optical interconnects and/or optical computing work. Lasers with submilliampere threshold currents will meet these requirements since with a modest current pulse $(\sim 30 \mathrm{~mA})$ they can be modulated at high speed without substantial delay between the application of a current puise and the onset of lasing. Buried heterostructure graded-index separate-confinement heterostructure single quanturn well (BH GRIN. SCH SQW ) lasers are capable of meeting these requirements as recently demonstrated.,

The suitability of BH GRIN-SCH SQW lasers for submiliampere operation can easily be seen by considering the threshold current density $J_{\mathrm{ts}},{ }^{3}$

$$
J_{\mathrm{th}}=J_{0}+\frac{\alpha d e}{B \tau \Gamma}+\frac{d e}{2 L B \tau \Gamma} \ln \frac{1}{R_{1} R_{2}},
$$

where $J_{o}$ is the transparency current density, $\alpha$ is the internal loss (active region and waveguiding), $d$ is the active region thickness, $e$ is the absolute value of the electron charge, $\tau$ is the effective recombination time near transparency, $B$ is the differential gain constant, $\Gamma$ is the optical confinement factor, $L$ is the laser cavity length, and $R_{1}$ and $R_{2}$ are the end facet refiectivities. In a GRIN-SCH SQW $\alpha$ can be expected

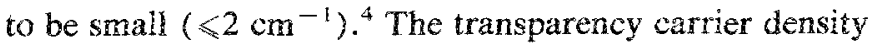
for a SQW is nearly the same as that for a conventional double heterostructure $(\mathrm{DH}){ }^{5}$ but the active region volume is much smaller in a SQW than in a DH so $J_{0}$ is much smaller for a SQW. This means that a SQW with appropriate optical confinement will have a much lower threshold than a $\mathrm{DH}$; furthermore, decreasing the end losses will lead to a much more significant reduction in threshold current for a SQW than for a DH laser, because the end losses account for a much greater fraction of the threshold gain for the $\mathrm{SQW}$. We can take advantage of this experimentally by evaporating high reflectivity dielectric coatings on the cleaved facets of GRIN-SCH SQW lasers to reduce the end losses. In this article we report on extremely high quality molecular-beam epitaxy (MBE) growth of GRIN-SCH SQW lasers necessary for improvement in BH GRIN-SCH SQW lasers and report on results with $B H$ GRIN-SCH SQW lasers with high refiectivity coatings.

GRIN-SCH SQW structures were grown on (100) GaAs substrates doped with Si to a level of about $5 \times 10^{17} \mathrm{~cm}^{-3}$. After the standard wafer preparation and oxide desorption, a $1.5-\mu \mathrm{m}$-thick buffer layer of $n-\mathrm{GaAs}$ was grown at $600^{\circ} \mathrm{C}$ followed by a $1.5-\mu \mathrm{m}$-thick $n-\mathrm{Al} 0.5 \mathrm{Ga}_{0.5} \mathrm{As}$ cladding layer grown at $700^{\circ} \mathrm{C}$. (In some samples a five period short period superlattice was grown between the buffer layer and cladding layers to impede impurity difusion.) The n-AlGaAs was graded in composition from $50 \%$ to $20 \%$ AlAs over $1750 \AA$ before the growth of a SQW with a thickness that varied between 40 and $100 \AA$. Following the quantum well, a p-A1GaAs layer graded from $20 \%$ to $50 \%$ AlAs over $1750 \AA$ was grown, and was capped with a $1.5 \mu \mathrm{m} p-\mathrm{Al}_{0.5} \mathrm{Ga}_{0.5} \mathrm{As}$ cladding layer. A $0.5 \mu \mathrm{m} p^{+}-\mathrm{GaAs}$ layer was then grown on top to facilitate contact formation. A schematic diagram of the structure is shown in Fig. 1.

Broad area lasers ( $110 \mu \mathrm{m}$ wide) from structures with quantum well thicknesses of $40,60,80$, and $100 \AA$ were fabricated and tested for their spectrum and current-light characteristics. Cavity lengths ranging from $\sim 500$ to $3000 \mu \mathrm{m}$ were examined. The best results were achieved for a $520-\mu \mathrm{m}$ long device with an $80-\mathrm{A}$-wide quanturn well which had a threshold current density of $93 \mathrm{~A} / \mathrm{cm}^{2}$. Table I details the laser threshold current densities for long (longer than 3 $\mathrm{mm}$ ) devices obtained from the onset of lasing emission atop spontaneous emission. In the range of lengths (3.09-3.29 $\mathrm{mm}$ ) examined, length differences should not affect the threshold current density appreciably, while with shorter devices $(400-600 \mu \mathrm{m})$ length differences would change 


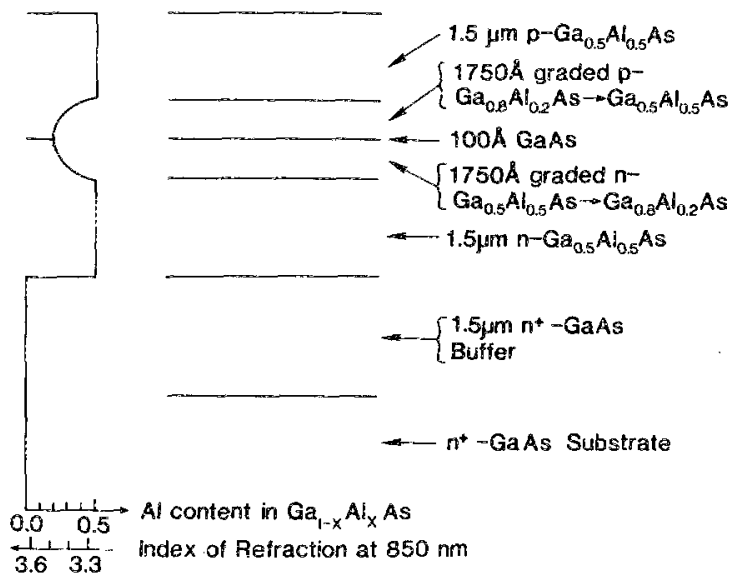

FiG. 1. Schematic diagram of a GRIN-SCH SQW laser showing layers and their indexes of refraction.

threshold current density significantiy. Therefore with long devices we can determine the dependence of threshold current density on quantum well width more easily. A striking observation is that the threshold current density is not appreciably dependent on the quantum well thickness in the range studied. This is attributed to the optical modal gain being proportional to the quantum well thickness, whereas the quantum well medium gain is inversely proportional to the quantum well size. The results indicate that these two effects nearly cancel each other over a certain range. ${ }^{6}$ In the limit of very thin $(<20 \AA)$ and very wide $(>200 \AA)$ quantum wells however, substantially higher threshold currents are expected, due to loss of carrier confinement in the former and the onset of three-dimensional density of states in the latter.

Some of the GRIN-SCH $100 \AA$ SQW structures were then used to fabricate buried lasers. Broad area devices $450 \mu \mathrm{m}$ long and $100 \AA$ wide fabricated from this earlier material had threshold current densities of $\sim 450 \mathrm{~A} / \mathrm{cm}^{2}$. Mesas 2 $\mu \mathrm{m}$ wide were etched through the MBE layers down to the substrate. A $1 \mu \mathrm{m} p-\mathrm{Al}_{0.3} \mathrm{Ga}_{0.7}$ As layer and a $3 \mu \mathrm{m} n$ $\mathrm{Al}_{0.3} \mathrm{Ga}_{0.7} \mathrm{As}$ layer were grown by liquid phase epitaxy (LPE) to provide current confinement. A shallow $\mathrm{Zn}$ diffusion was performed to facilitate formation of an Ohmic contact on the $p$ side of the device, and Ohmic contacts were applied. Finished devices had an active layer stripe width of $1 \mu \mathrm{m}$. These devices were found to have average internal losses (including waveguiding losses) of $\sim 13 \mathrm{~cm}^{-1}$.

The modal gain $g$ for a semiconductor laser is given by

$$
g=\alpha+\frac{1}{2 L} \ln \frac{1}{R_{1} R_{2}} .
$$

Using this relationship, the measured value of the loss, and

TABLE I. Laser characteristics vs quantum well width.

\begin{tabular}{rccr}
\hline Well width & Cavity length & Lasing wavelength & \multicolumn{1}{c}{$J_{\mathrm{th}}$} \\
\hline $100 \AA$ & $3.29 \mathrm{~mm}$ & $8702 \pm 30 \AA$ & $115 \mathrm{~A} / \mathrm{cm}^{2}$ \\
$80 \AA$ & $3.21 \mathrm{~mm}$ & $8640 \pm 25 \AA$ & $93 \AA / \mathrm{cm}^{2}$ \\
$60 \AA$ & $3.09 \mathrm{~mm}$ & $8578 \pm 21 \AA$ & $120 \mathrm{~A} / \mathrm{cm}^{2}$ \\
$40 \AA$ & $3.24 \mathrm{~mm}$ & $8363 \pm 24 \AA$ & $117 \mathrm{~A} / \mathrm{cm}^{2}$ \\
\hline
\end{tabular}
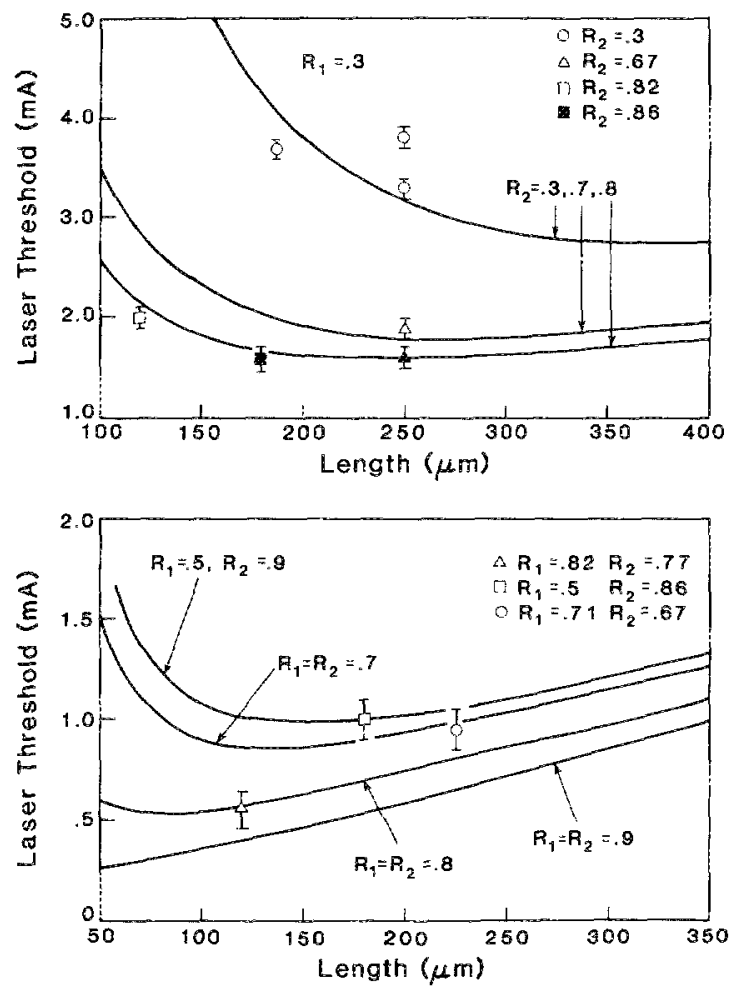

FIG. 2. Calculated threshold current for a BH GRIN-SCH SQW laser with various cavity lengths and facet reflectivities. Experimental points are also shown.

the gain versus current relationship calculated in the manner of Arakawa and Yariv, ${ }^{7}$ expected values of threshold current for various cavity lengths and facet reflectivities were calculated. These theoretical results are shown in Fig. 2. Experimental results for cavity lengths of 250,180 , and $120 \mu \mathrm{m}$ are also shown in Fig. 2. Threshold currents were measured $\mathrm{cw}$ at room temperature. The most dramatic threshold reductions with high reflectivity coatings were obtained for short lasers since end losses are more significant for shorter cavities. As shown in Fig. 3(b) with no high reflectivity coatings a $120-\mu \mathrm{m}$-long laser had a threshoid of $5.5 \mathrm{~mA}$. When both end facets were coated to $\sim 80 \%$ reflectivity the threshold current for the same laser was reduced to $0.55 \mathrm{~mA}$ as shown in Fig. 3(a). This value represents a substantial benchmark for lasers intended for optical computing.

The delay time for the onset of lasing following a current pulse of amplitude $I$ assuming a constant carrier lifetime $\tau_{s}$ is given $b y^{8}$

$$
\tau_{d}=\tau_{s} \ln \left(\frac{I}{I-I_{\mathrm{th}}}\right) \rightarrow \tau_{s} \frac{I_{\mathrm{th}}}{I},
$$

for large $I$, where $I_{\text {th }}$ is the cw threshold current. Clearly lasers with small $I_{\text {th }}$ will have shorter delay times for pulses of equal amplitude. We have measured the delay time for a BH GRIN-SCH SQW laser with a cavity length of $250 \mu \mathrm{m}$, $\sim 70 \%$ reflectivity coatings on both facets and a cw threshold current of $0.95 \mathrm{~mA}$. A direct pulse current without bias was applied to the laser. The drive pulse amplitude was controlled by a broadband continuously variable rf attenuator. The pulse shape was maintained in all measurements. To accurately measure the switch-on delay down to $<50$ ps 


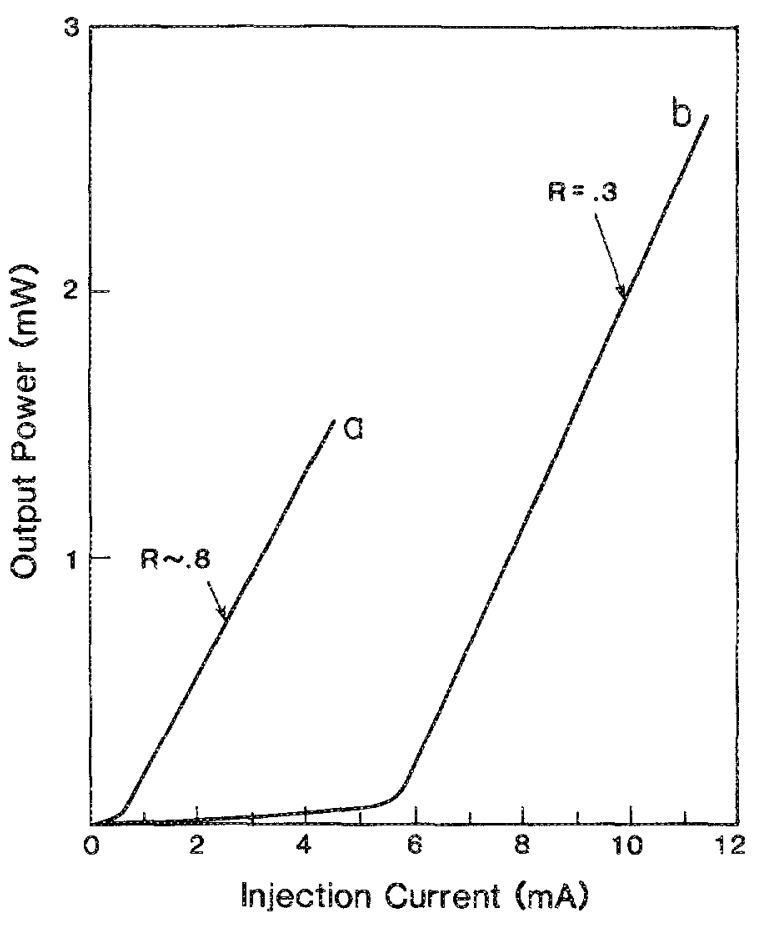

Frg. 3. Light output us current curve for a $120 \mu m$-long BH GRIN-SCH SQW laser with (a) high reflectivity coated cleaved facets and (b) uncoated cleaved facets. Both curves are for the same laser.

time scale it was assumed that the laser turns off without delay at the termination of the current pulse; this assumption was well supported by the observed data. The results, shown in Fig. 4, demonstrate that the laser had a switch-on delay of between 20 and 50 ps with a modest $30 \mathrm{~mA}$ pulse drive.

In cases where a laser is modulated by a real digital pulse stream, the starting condition for each pulse is affected by the charge leftover from the previous pulse if one existed. 9,10

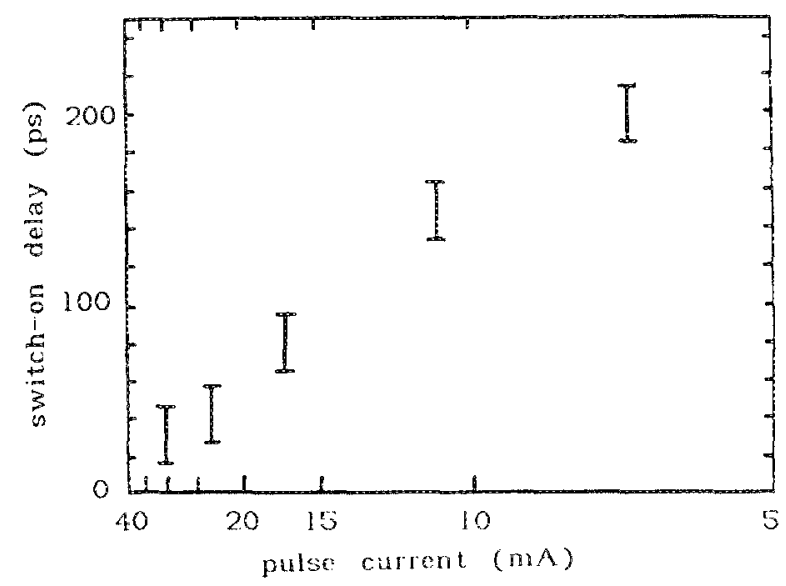

FIG. 4. Measured switch-on delay vs pulse current amplitude for a $250-\mu \mathrm{m}$ long BH GRIN-SCH SQW with $\sim 70 \%$ reflectivity facet coatings and a $\mathrm{cw}$ threshold current of $0.95 \mathrm{~mA}$.
The charge density typically stays at slightly below the threshold level following an optical pulse, and decays with a time constant equal to the carrier lifetime. The second of two consecutive optical pulses will have a higher amplitude; this is called the pattern effect. When driven by two consecutive current pulses separated by as little as 200 ps (limited by the rise time of the pulser) the present BH GRIN-SCH SQW lasers, however, showed no pattern effect.

In conclusion, we have demonstrated very low current density broad area lasers which can be applied to fabricating submilliampere buried GRIN-SCH SQW. We have fabricated PH GRIN-SCH SQW (A1,Ga)As lasers with high reflectivity coatings which have $\mathrm{cw}$ threshold currents as low as $0.55 \mathrm{~mA}$. We have also demonstrated that these lasers can be modulated directly at high speed with logic-level drives without the need for current prebias. The lasers exhibit truly on-off switching with switch-on delays of $<50 \mathrm{ps}$; the patiern effect is eliminated. Since our best GRIN-SCH SQW lasers were not used for making buried lasers and the regrowth was not optimized for limiting current leakage (which was significant) we expect that threshold currents as low as $0.2 \mathrm{~mA}$ should be realizable with optimized devices.

Acknowledgments: The Caltech portion of this work was supported by the National Science Foundation, the Office of Naval Research, and the Air Force Office of Scientific Research. H. M. was partially supported by SDIO-IST through the Jet Propulsion Laboratory. The ORTEL portion was supported by the Defense Advanced Research Projects Agency under the Optical Computing Program and Naval Research Laboratory.

\footnotetext{
a) On leave from the University of Illinois at Urbana-Champaign, Coordinated Science Laboratory, 1101 West Springfield Avenue, Urbana, Illinois 61801 .

${ }^{1}$ P. L. Derry, A. Yariv, K. Y. Lau, N. Bar-Chaim, K. Lee, and J. Rosenberg, Appl. Phys. Lett. 50, 1773 (1987).

${ }^{2}$ K. Y. Lau, N. Bar-Chaim, P. L. Derry, and A. Yariv, Appl. Phys. Lett. 51, $69(1987)$

${ }^{3}$ H. C. Casey, Jr. and M. B. Parish, Heterostructure Lasers Part A: Fundamental Principles (Academic, Orlando, 1978), p. 183.

'M. Mittelstein, Y. Arakawa, A. Larsson, and A. Yariv, Appl. Phys. Lett. 49, 1689 (1986).

${ }^{5}$ M. Mittelstein, A. Larsson, and A. Yariv (unpublished results).

${ }^{\circ} \mathrm{H}$. Z. Chen, A. Ghaffari, $\mathrm{H}$. Morkoç, and A. Yariv, Appl. Phys. Lett. 25, 2094 (1987).

${ }^{7}$ Y. Arakawa and A. Yariv, IEEE J. Quantum Eleciron. 21, 1666 (1985). ${ }^{8} \mathrm{H}$. C. Casey, Jr. and M. B. Panish, Heterostructure Lasers Part $B$ : Materials and Operaing Characteristics (Academic, Orlando, 1978), p. 228.

${ }^{\circ}$ P. M. Boers and M. T. Vlaadingerbroek, Electron. Lett. 11, 206 (1975).

$10 \mathrm{~J}$. E. Bowers, B. R. Hemenway, A. H. Gnauck, and D. P. Wilt, IEEE J. Quantum Electron. 22, 833 (1986).
} 